PROCEEDINGS OF THE

AMERICAN MATHEMATICAL SOCIETY

Volume 127, Number 9, Pages 2735-2744

S 0002-9939(99)05280-6

Article electronically published on April 23, 1999

\title{
FUNDAMENTAL THEOREM OF GEOMETRY WITHOUT THE 1-TO-1 ASSUMPTION
}

\author{
ALEXANDER CHUBAREV AND IOSIF PINELIS
}

(Communicated by Christopher Croke)

\begin{abstract}
It is proved that any mapping of an $n$-dimensional affine space over a division ring $\mathbb{D}$ onto itself which maps every line into a line is semiaffine, if $n \in\{2,3, \ldots\}$ and $\mathbb{D} \neq \mathbb{Z}_{2}$. This result seems to be new even for the real affine spaces. Some further generalizations are also given. The paper is self-contained, modulo some basic terms and elementary facts concerning linear spaces and also - if the reader is interested in $\mathbb{D}$ other than $\mathbb{R}, \mathbb{Z}_{p}$, or $\mathbb{C}$ - division rings.
\end{abstract}

\section{TERMINOLOGY AND NOTATION}

Let $\mathbb{D}$ be a division ring and let $\mathcal{L}$ be a finite-dimensional linear space over $\mathbb{D}$. An affine space [in $\mathcal{L}$ ] over $\mathbb{D}$ is defined here simply as any set of the form $\mathcal{A}:=P+\Lambda$, where $P \in \mathcal{L}$ and $\Lambda$ is a linear subspace of $\mathcal{L}$. Given $\mathcal{L}$ and $\mathcal{A}$, the linear subspace $\Lambda$ is uniquely determined; let us then put $\operatorname{Tan} \mathcal{A}:=\Lambda$ and $\operatorname{dim} \mathcal{A}:=\operatorname{dim} \operatorname{Tan} \mathcal{A}$. If $\mathcal{A}$ and $\Pi$ are affine spaces [in $\mathcal{L}$ ] over $\mathbb{D}$ and $\Pi \subseteq \mathcal{A}$, then $\Pi$ is called an affine subspace of $\mathcal{A}$. For brevity, let us refer to a $k$-dimensional affine space over $\mathbb{D}$ as a $k$-plane; then, 1-planes will be referred to simply as lines. In what follows, $\mathcal{A}$ is an affine space, and capital Roman letters, possibly with subscript indices, stand for elements of $\mathcal{A}$, unless otherwise specified. Let us denote the line through $P$ and $Q$ by $P Q$, and this notation will imply that $P$ and $Q$ are assumed or have been proved to be distinct or that this is not difficult to see. For any non-empty set $\mathcal{E} \subseteq \mathcal{A}$, let aff $\mathcal{E}$ denote the intersection of all the affine subspaces of $\mathcal{A}$ containing $\mathcal{E}$; note that aff $\mathcal{E}$ is an affine subspace of $\mathcal{A}$ [R.1]. Here and in what follows, [R.\#] refers to Remark \#; such remarks, providing additional details, have been placed at the end of the paper so as not to distract the reader from the main course unnecessarily. For any $\mathcal{E} \subseteq \mathcal{A}$, let $\operatorname{dim} \mathcal{E}:=\operatorname{dim}$ aff $\mathcal{E} ; \operatorname{dim} \emptyset:=-1$; one always has $\operatorname{dim} \mathcal{E} \leq$ card $\mathcal{E}-1$; if $\operatorname{dim} \mathcal{E}=\operatorname{card} \mathcal{E}-1=k, \mathcal{E}$ is called a $k$-simplex; if $\mathcal{E}$ is a $k$-simplex for some $k$, it is called a simplex. It will be convenient for us to say that two $k$-planes $\pi_{1}$ and $\pi_{2}$ are parallel and to write $\pi_{1} \| \pi_{2}$ if $\pi_{1} \cap \pi_{2}=\emptyset$ and $\operatorname{dim}\left(\pi_{1} \cup \pi_{2}\right)=k+1$; this is a non-reflexive version of the notion of parallelism. By a parallelogram, we mean a 4-tuple $\left(P_{0}, P_{1}, P_{2}, P_{3}\right)$ such that $P_{3}-P_{0}=P_{2}-P_{1}$ and $\operatorname{dim}\left\{P_{0}, P_{1}, P_{2}, P_{3}\right\}=2$;

Received by the editors June 21, 1996.

1991 Mathematics Subject Classification. Primary 51A15; Secondary 51A05, 51A45, 51A25, 51D15, 51D30, 51E15, 51N10, 51N15, 14P99, $05 \mathrm{~B} 25$.

Key words and phrases. Fundamental theorem of geometry, affine space, affine transformation, semi-affine transformation, collineation, isomorphism, parallelism, incidence relations, projective transformation. 
equivalently, $\left(P_{0}, P_{1}, P_{2}, P_{3}\right)$ is a parallelogram iff $P_{0} P_{1} \| P_{2} P_{3}$ and $P_{0} P_{3} \| P_{1} P_{2}$ [R.7].

In the sequel, let $\mathcal{A}$ and $\mathcal{A}^{\prime}$ be affine spaces of finite dimensions $n$ and $n^{\prime}$ over division rings $\mathbb{D}$ and $\mathbb{D}^{\prime}$, respectively, and let $T$ be a mapping of $\mathcal{A}$ into $\mathcal{A}^{\prime}$.

Let $P^{\prime}:=T(P)$ for all $P \in \mathcal{A}$.

Let us say that $T$ is $f$-semi-affine, for some isomorphism $f: \mathbb{D} \rightarrow \mathbb{D}^{\prime}$, if

$$
T\left(P_{0}+\alpha\left(P_{1}-P_{0}\right)+\beta\left(P_{2}-P_{0}\right)\right)=P_{0}^{\prime}+f(\alpha)\left(P_{1}^{\prime}-P_{0}^{\prime}\right)+f(\beta)\left(P_{2}^{\prime}-P_{0}^{\prime}\right)
$$

for all $\left(P_{0}, P_{1}, P_{2}\right) \in \mathcal{A}^{3}$ and all $(\alpha, \beta) \in \mathbb{D}^{2}$. If here $\mathbb{D}=\mathbb{D}^{\prime}$ and $f$ is the identity mapping, then $T$ is called affine. [Note that $T$ is affine iff the mapping $P-P_{0} \mapsto$ $P^{\prime}-P_{0}^{\prime}$ is linear for some or, equivalently, for any fixed $P_{0}$.] Let us call $T$ semi-affine if it is $f$-semi-affine for some isomorphism $f$ [R.8].

\section{INTRODUCTION}

The fundamental theorem of [projective] geometry goes back to von Staudt (1847); see, e.g., [4, page 38]. It has since appeared in different forms and in different degrees of generality, mostly in textbooks and monographs rather than in articles. Using the notion of the points at infinity, one of the modern versions of the fundamental theorem of projective geometry [1, page 88] can be interpreted in terms of affine geometry as

Theorem P. Suppose that $n^{\prime}=n \geq 2, T$ is 1 -to-1 and onto and maps every line in $\mathcal{A}$ onto a line, and the images of any two parallel lines in $\mathcal{A}$ under $T$ are parallel lines. Then $T$ is semi-affine.

The name $\mathrm{P}$ is given here to this theorem to allude to its "projective" origin. Variants of this theorem can be found, e.g., in [9] for free modules over [commutative] local rings.

Advantages of projective geometry over its affine counterpart, foremost the duality between points and lines, are well known. Nonetheless, the non-reflexive notion of parallelism, lost or at most implicit in projective geometry, may still be quite useful. For instance, retaining only the last portion of the proof of Theorem (main) that begins right after the proof of Lemma 5 and referring to the parallelismpreservation and line-onto-line conditions of Theorem $\mathrm{P}$ rather than to Lemmas 5 and 4, one obtains a self-contained half-page proof of Theorem P. The same proof remains valid for the following, streamlined, version of Theorem $\mathrm{P}$, in which the restrictions that $n^{\prime}=n$ and $T$ is 1 -to- 1 and onto are absent.

Theorem P1. Suppose that $n \geq 2$ and that $T$ maps every line in $\mathcal{A}$ onto a line [R.10] and every parallelogram in $\mathcal{A}$ onto a parallelogram. Then $T$ is semi-affine.

Thus, the parallelism preservation is a very strong restriction. The same is true regarding the 1-to-1 condition.

In this paper, we shall show that in Theorem $\mathrm{P}$, the 1-to-1 and parallelism preservation conditions can both be removed and the line-onto-line condition replaced by the weaker line-into-line or, even more generally, $q$-plane-into- $q$-plane condition, at least if $\mathbb{D} \neq \mathbb{Z}_{2}$. In addition, the restriction $n^{\prime}=n$ of Theorem $\mathrm{P}$ may be relaxed to $n^{\prime} \geq n$. 


\section{StATEMENT AND DISCUSSION OF MAIN RESULTS}

Theorem (main). Let $\mathbb{D} \neq \mathbb{Z}_{2}$ and $n^{\prime} \geq n \geq 2$. Suppose that $T$ is onto and maps every $q$-plane in $\mathcal{A}$ into a q-plane, for some $q \in\{1, \ldots, n-1\}$. Then $T$ is semi-affine.

In particular, this theorem implies that, a posteriori, $\mathbb{D}$ and $\mathbb{D}^{\prime}$ are isomorphic and $n^{\prime}=n$.

The conditions of Theorem (main) are minimal in the sense that none of them:

(i) $\mathbb{D} \neq \mathbb{Z}_{2}$,

(ii) $n^{\prime} \geq n$,

(iii) $q \in\{1, \ldots, n-1\}$ [whence, with necessity, $n \geq 2$ ],

(iv) $T$ being onto,

(v) $T$ mapping $q$-planes into $q$-planes

may be dropped [R.11]. Of course, this does not mean that these conditions cannot be relaxed in any way; for instance, Proposition 1 below complements Theorem (main) in some cases in which $\mathbb{D}=\mathbb{Z}_{2}$.

Choosing $q=1$ in Theorem (main), one has

Corollary 1. Let $\mathbb{D} \neq \mathbb{Z}_{2}$ and $n^{\prime} \geq n \geq 2$. Suppose that $T$ is onto and maps every line in $\mathcal{A}$ into a line. Then $T$ is semi-affine.

Corollary 2. Let $n^{\prime} \geq n \geq 2$ and $\mathbb{D}^{\prime}=\mathbb{D}=\mathbb{Q}, \mathbb{R}$, or $\mathbb{Z}_{p}$ for some prime $p>2$. Suppose that $T$ is onto and maps every q-plane in $\mathcal{A}$ into a q-plane, for some $q \in\{1, \ldots, n-1\}$. Then $T$ is affine.

Corollary 2 is also immediate from Theorem (main), because the only automorphisms of $\mathbb{Q}, \mathbb{R}$, or $\mathbb{Z}_{p}$ are the identity mappings.

With the additional conditions that $\mathbb{D}=\mathbb{D}^{\prime}=\mathbb{R}, n^{\prime}=n$, and $T$ is 1 -to- 1 and maps every line onto a line, a variant of Corollary 1 is stated as the Fundamental Theorem of Affine Geometry in [7, page 925].

Another result similar to Corollary 1 is stated in [3] as Theorem 2.6.3, with the additional conditions that $T$ is 1 -to- $1, \mathbb{D}$ and $\mathbb{D}^{\prime}$ are commutative, and $n^{\prime}=n$; the condition $\mathbb{D} \neq \mathbb{Z}_{2}$ seems to be missing there, which makes the claim incorrect [R.12]. As to the proof suggested in [3], in its Step 2, in the terms used therein, $f(D)$ and $D^{\prime}$ are confused, and to use the implication $f(D) \cap f\left(D^{\prime}\right)=\emptyset \Longrightarrow f(D) \| f\left(D^{\prime}\right)$, one ought first to prove that every 2-plane is mapped into a 2-plane, and it is here that the condition $\mathbb{D} \neq \mathbb{Z}_{2}$ is needed - cf. Remark 13 or the proof of Lemma 2 ( $k$-plane-into- $k$-plane) below.

With the additional conditions that $T$ is 1 -to- $1, \mathbb{D}=\mathbb{D}^{\prime}$, and $n^{\prime}=n$, Corollary 1 is stated in [6] as Theorem 2 ; the condition $\mathbb{D} \neq \mathbb{Z}_{2}$ seems to be missing there as well, and the proof for the case $n \geq 3$, where the condition $\mathbb{D} \neq \mathbb{Z}_{2}$ is actually needed [R.12], is left to the reader.

For the commutative $\mathbb{D}$ and $\mathbb{D}^{\prime}$, Corollary 1 is stated also in [5] on page 93 . The very first line of the proof suggested therein, translated into the terms used in this paper, says, "By supposition, $T(\operatorname{aff}\{P, Q\}) \subseteq$ aff $\left\{P^{\prime}, Q^{\prime}\right\}$ "; we however find this to be supposed or immediately following the supposition only if $P^{\prime} \neq Q^{\prime}$ or $P=Q$; in the remaining case when $P \neq Q$ but $P^{\prime}=Q^{\prime}$, we do not know how to prove this inclusion [or rather that $T$ is actually 1-to-1, so that the latter case is in fact impossible] significantly simpler than just to repeat most of the proof below of Theorem (main), namely, Lemma 2 through Lemma 5. In our opinion, 
the following additional assumption is in fact used in [5]: if the images under $T$ of two distinct points coincide, then the image of the entire line through the two points contains only one point; such an additional assumption, while completely unnecessary, is effectively as strong as the 1-to-1 assumption and would simplify the matter greatly [R.13].

For $n=n^{\prime}=2$ and commutative $\mathbb{D}=\mathbb{D}^{\prime}$, an analogue of the fundamental theorem of geometry for $T$ defined on a subset of the affine space is given in [2] (as Theorem 1 of Schaeffer, on page 92 therein) with $T$ assumed to be 1-to-1 only at certain points (in a certain sense), rather than on its entire domain of definition. One can also find in [2] many other analogues of the fundamental theorem of geometry, mostly under the 1-to-1 assumption.

The case $\mathbb{D}=\mathbb{Z}_{2}$, although very simple to analyze, is peculiar [R.12, R.9]. The following proposition complements Theorem (main).

Proposition 1. Let $\mathbb{D}^{\prime}=\mathbb{D}=\mathbb{Z}_{2}, n^{\prime} \geq n \geq 2, q \in\{1, \ldots, n-1\}$, and $n=2$ or $q \geq 2$. Suppose that $T$ is onto and maps every q-plane in $\mathcal{A}$ into a q-plane. Then $T$ is affine.

The following corollaries are free of any restrictions on the division rings. They are immediate from Theorem (main) and Proposition 1.

Corollary 3. Suppose that $n^{\prime} \geq n \geq 3$ and $T$ is onto and maps every q-plane in $\mathcal{A}$ into a q-plane, for some $q \in\{2, \ldots, n-1\}$. Then $T$ is semi-affine.

Corollary 4. Suppose that $n^{\prime} \geq n \geq 2$ and $T$ is onto and maps every $(n-1)$-plane in $\mathcal{A}$ into an $(n-1)$-plane. Then $T$ is semi-affine.

\section{Proofs}

The proof of Theorem (main) is based on the following lemmas, in which all the conditions of Theorem (main) are assumed to hold.

Lemma 1 (lowering-plane-preservation-dimension). If $T$ maps every $k$-plane in $\mathcal{A}$ into a $k$-plane for some $k \in\{1, \ldots, n-1\}$, then $T$ maps every $(k-1)$-plane in $\mathcal{A}$ into a $(k-1)$-plane.

Proof. Let $\pi$ be any $(k-1)$-plane in $\mathcal{A}$. Choose arbitrarily a $k$-plane $\Pi_{1}$ in $\mathcal{A}$, containing $\pi$ [R.2]. Then there exists a $k$-plane $\Pi_{1}^{\prime}$ in $\mathcal{A}^{\prime}$ such that $T\left(\Pi_{1}\right) \subseteq \Pi_{1}^{\prime}$. Because $T$ is onto and $\operatorname{dim} \Pi_{1}^{\prime}=k \leq n-1<n^{\prime}$, there exists a point $A \in \mathcal{A}$ such that $A^{\prime} \notin \Pi_{1}^{\prime}$. Then $A \notin \Pi_{1}$, and so, $A \notin \pi$. Introduce $\Pi_{2}:=\operatorname{aff}(\pi \cup\{A\})$. Then $\Pi_{2}$ is a $k$-plane [R.2]; also, $\pi \subseteq \Pi_{1} \cap \Pi_{2}$. Next, there exists a $k$-plane $\Pi_{2}^{\prime}$ in $\mathcal{A}^{\prime}$ such that $T\left(\Pi_{2}\right) \subseteq \Pi_{2}^{\prime}$. Then $A^{\prime} \in T\left(\Pi_{2}\right) \backslash \Pi_{1}^{\prime} \subseteq \Pi_{2}^{\prime} \backslash \Pi_{1}^{\prime}$, and so, $\Pi_{2}^{\prime} \neq \Pi_{1}^{\prime}$, whence $\operatorname{dim}\left(\Pi_{1}^{\prime} \cap \Pi_{2}^{\prime}\right) \leq k-1$ [R.3]. Finally, $T(\pi) \subseteq T\left(\Pi_{1}\right) \cap T\left(\Pi_{2}\right) \subseteq \Pi_{1}^{\prime} \cap \Pi_{2}^{\prime}$. Therefore, $\operatorname{dim} T(\pi) \leq k-1$.

Lemma 2 ( $k$-plane-into- $k$-plane). $T$ maps every $k$-plane in $\mathcal{A}$ into a $k$-plane, for every $k \in\{0, \ldots, n\}$.

Proof. Assume that, on the contrary, there exists $k \in\{1, \ldots, n\}$ such that $T$ maps every $j$-plane in $\mathcal{A}$ into a $j$-plane, for every $j \in\{0, \ldots, k-1\}$, but there exists a $k$-plane $\Pi$ in $\mathcal{A}$ such that $\operatorname{dim} T(\Pi) \geq k+1$. Then one must have $k \geq 2$, in view of Lemma 1 (lowering-plane-preservation-dimension) and the condition $q \geq 1$ of Theorem (main). Since $\operatorname{dim} T(\Pi) \geq k+1$, there exists a $(k+1)$-simplex $\mathcal{E}^{\prime}$ in $T(\Pi)$; hence, there exists a set $\mathcal{E}:=\left\{P_{0}, \ldots, P_{k+1}\right\} \subset \Pi$ such that $T(\mathcal{E})=\mathcal{E}^{\prime}$, and so, 
$\operatorname{dim} T(\mathcal{E})=k+1$. Without loss of generality [w.l.o.g.], $P_{k+1}=\alpha_{0} P_{0}+\cdots+\alpha_{k} P_{k}$, for some $\left(\alpha_{0}, \ldots, \alpha_{k}\right) \in \mathbb{D}^{k+1}$ such that $\alpha_{0}+\cdots+\alpha_{k}=1$ [R.14].

If $\exists i \in\{0, \ldots, k\} \alpha_{i} \neq 1$, say $\alpha_{0} \neq 1$, then, introducing $\alpha:=\left(1-\alpha_{0}\right)^{-1}$, one has $(1-\alpha) P_{0}+\alpha P_{k+1}=\alpha \alpha_{1} P_{1}+\cdots+\alpha \alpha_{k} P_{k}$ and $\alpha \alpha_{1}+\cdots+\alpha \alpha_{k}=1$; hence, aff $\left\{P_{0}, P_{k+1}\right\} \cap$ aff $\left\{P_{1}, \ldots, P_{k}\right\} \neq \emptyset$.

Otherwise, $\alpha_{0}=1, \ldots, \alpha_{k}=1$ [one might note here that this, in conjunction with $\alpha_{0}+\cdots+\alpha_{k}=1$, may be a possibility only if the characteristic of $\mathbb{D}$ divides $k]$. Then introduce $Q:=(1-\beta) P_{0}+\beta P_{1}$, for some $\beta \in \mathbb{D} \backslash\{0,1\}$; such a $\beta$ exists, since $\mathbb{D} \neq \mathbb{Z}_{2}$ [this is the only place in the proof of Theorem (main) where the restriction on $\mathbb{D}$ is used]. Set $\mathcal{F}:=\left\{Q_{0}, \ldots, Q_{k+1}\right\}$, where $Q_{0}:=Q, Q_{j}:=P_{j}$ for $j \in\{2, \ldots, k+1\}, Q_{1}:=P_{0}$ if $Q^{\prime} \neq P_{0}^{\prime}$, and $Q_{1}:=P_{1}$ if $Q^{\prime}=P_{0}^{\prime}$. Note that $Q^{\prime}=P_{0}^{\prime}$ implies $Q^{\prime} \neq P_{1}^{\prime}$, because $\operatorname{dim} T(\mathcal{E})=k+1$ implies that $P_{0}^{\prime}, P_{1}^{\prime}, \ldots, P_{k+1}^{\prime}$ are all distinct. Hence, w.l.o.g., $Q^{\prime} \neq P_{0}^{\prime}$. Thus, $Q_{1}=P_{0}$. Recall that $\alpha_{0}=\cdots=\alpha_{k}=1$, whence $P_{k+1}=P_{0}+\cdots+P_{k}$; therefore, $Q_{k+1}=\beta_{0} Q_{0}+\cdots+\beta_{k} Q_{k}$, where $\beta_{0}:=\beta^{-1}$, $\beta_{1}:=2-\beta^{-1}, \beta_{j}:=1$ for $j \in\{2, \ldots, k+1\}$. Hence, similarly to the above relation for the set $\mathcal{E}=\left\{P_{0}, \ldots, P_{k+1}\right\}$, one obtains $\operatorname{aff}\left\{Q_{0}, Q_{k+1}\right\} \cap$ aff $\left\{Q_{1}, \ldots, Q_{k}\right\} \neq \emptyset$, because $\beta_{0} \neq 1$. By the definition of the $Q_{j}$ 's, one has $P_{0}^{\prime}=Q_{1}^{\prime}, P_{j}^{\prime}=Q_{j}^{\prime}$ for $j \in\{2, \ldots, k+1\}$, and $P_{1}^{\prime} \in Q^{\prime} P_{0}^{\prime}=Q_{0}^{\prime} Q_{1}^{\prime}\left[\mathrm{R} .15\right.$; recall that $\left.Q^{\prime} \neq P_{0}^{\prime}\right]$. Hence, $T(\mathcal{E}) \subset$ aff $T(\mathcal{F})$, and so, $\operatorname{dim} T(\mathcal{F})=\operatorname{dim}$ aff $T(\mathcal{F}) \geq \operatorname{dim} T(\mathcal{E})=k+1$.

Thus, in any case, one can find a set $\mathcal{G}:=\left\{R_{0}, \ldots, R_{k+1}\right\} \subset \Pi$ such that $\operatorname{dim} T(\mathcal{G}) \geq k+1$ and aff $\left\{R_{0}, R_{k+1}\right\} \cap \operatorname{aff}\left\{R_{1}, \ldots, R_{k}\right\} \neq \emptyset$. Hence, it follows that $T\left(\operatorname{aff}\left\{R_{0}, R_{k+1}\right\}\right) \cap T\left(\operatorname{aff}\left\{R_{1}, \ldots, R_{k}\right\}\right) \neq \emptyset$. Note also that $\operatorname{dim} \operatorname{aff}\left\{R_{1}, \ldots, R_{k}\right\} \leq$ $k-1$. Consequently, $\operatorname{dim} T\left(\operatorname{aff}\left\{R_{1}, \ldots, R_{k}\right\}\right) \leq k-1$, as well as $\operatorname{dim} T\left(\operatorname{aff}\left\{R_{0}, R_{k+1}\right\}\right)$ $\leq 1$, since $T$ maps every $j$-plane in $\mathcal{A}$ into a $j$-plane for every $j \in\{0, \ldots, k-1\}$, and $k \geq 2$. Therefore [R.16],

$$
\begin{aligned}
\operatorname{dim} T(\mathcal{G}) & \leq \operatorname{dim}\left(T\left(\operatorname{aff}\left\{R_{0}, R_{k+1}\right\}\right) \bigcup T\left(\operatorname{aff}\left\{R_{1}, \ldots, R_{k}\right\}\right)\right) \\
& \leq \operatorname{dim} T\left(\operatorname{aff}\left\{R_{0}, R_{k+1}\right\}\right)+\operatorname{dim} T\left(\operatorname{aff}\left\{R_{1}, \ldots, R_{k}\right\}\right) \leq 1+(k-1)=k,
\end{aligned}
$$

a contradiction.

Lemma 3 (preimage-of- $(n-1)$-plane-is- $(n-1)$-plane). Let $\Pi^{\prime}$ be an $(n-1)$-plane in $\mathcal{A}^{\prime}$. Then $T^{-1}\left(\Pi^{\prime}\right)$ is an $(n-1)$-plane.

Proof. Let $\mathcal{E}^{\prime}$ be an $(n-1)$-simplex in $\Pi^{\prime}$. Since $T$ is onto, there exists a set $\mathcal{E} \subset \mathcal{A}$ such that $T(\mathcal{E})=\mathcal{E}^{\prime}$ and $\operatorname{card} \mathcal{E}=\operatorname{card} \mathcal{E}^{\prime}[=n]$. Set $\Pi:=\operatorname{aff} \mathcal{E}$. Then $\Pi$ is an $(n-1)$-plane; indeed, $\operatorname{dim} \Pi=\operatorname{dim} \mathcal{E} \leq \operatorname{card} \mathcal{E}-1=n-1$ and, on the other hand, $n-1=\operatorname{dim} \mathcal{E}^{\prime}=\operatorname{dim} T(\mathcal{E}) \leq \operatorname{dim} T(\Pi) \leq \operatorname{dim} \Pi$ [the last inequality follows by Lemma 2 ( $k$-plane-into- $k$-plane)]. Note also that $T(\Pi) \subseteq \Pi^{\prime}$; indeed, again by Lemma 2 ( $k$-plane-into- $k$-plane), there exists an $(n-1)$-plane $\tilde{\Pi}$ in $\mathcal{A}^{\prime}$ such that $T(\Pi) \subseteq \tilde{\Pi}$, and so, $\tilde{\Pi} \supset T(\mathcal{E})=\mathcal{E}^{\prime}$, whence $\tilde{\Pi}=\operatorname{aff}\left(\mathcal{E}^{\prime}\right)=\Pi^{\prime}$. Thus, $\Pi$ is an $(n-1)$-plane such that $\Pi \subseteq T^{-1}\left(\Pi^{\prime}\right)$.

It remains to show that $T^{-1}\left(\Pi^{\prime}\right) \backslash \Pi=\emptyset$. Let, on the contrary, $P \in T^{-1}\left(\Pi^{\prime}\right) \backslash \Pi$. Let $\Pi_{1}^{\prime}$ be any $(n-1)$-plane in $\mathcal{A}^{\prime}$ such that $\Pi_{1}^{\prime} \| \Pi^{\prime}$. One can construct, analogously to $\Pi$, an $(n-1)$-plane $\Pi_{1}$ in $\mathcal{A}$ such that $\Pi_{1} \subseteq T^{-1}\left(\Pi_{1}^{\prime}\right)$. Then $\Pi_{1} \| \Pi$, because $\Pi_{1} \cap \Pi \subseteq T^{-1}\left(\Pi_{1}^{\prime} \cap \Pi^{\prime}\right)=\emptyset$. Since card $\mathcal{E}^{\prime}=n \geq 2$ and $T(\mathcal{E})=\mathcal{E}^{\prime}$, there exists a point $Q \in \mathcal{E}$ such that $Q^{\prime} \neq P^{\prime}$. Then $Q \neq P$ and, once more by Lemma $2(k$ plane-into- $k$-plane), $T(P Q) \subseteq P^{\prime} Q^{\prime} \subseteq \Pi^{\prime}$. But there exists a point $A \in P Q \cap \Pi_{1}$, because $\Pi_{1} \| \Pi, P \notin \Pi$, and $Q \in \Pi[R .6]$. Thus, $A^{\prime} \in P^{\prime} Q^{\prime} \cap T\left(\Pi_{1}\right) \subseteq \Pi^{\prime} \cap \Pi_{1}^{\prime}=\emptyset$, a contradiction. 
Lemma 4 ( $k$-plane-onto- $k$-plane). $T$ maps every $k$-plane in $\mathcal{A}$ onto a $k$-plane, for every $k \in\{0, \ldots, n\}$.

Proof. Let $\Pi$ be any $(n-1)$-plane in $\mathcal{A}$. By Lemma 2 ( $k$-plane-into- $k$-plane), there exists an $(n-1)$-plane $\Pi^{\prime}$ in $\mathcal{A}^{\prime}$ such that $T(\Pi) \subseteq \Pi^{\prime}$. Hence, by Lemma 3 (preimageof- $(n-1)$-plane-is- $(n-1)$-plane), $T^{-1}\left(\Pi^{\prime}\right)$ is an $(n-1)$-plane containing $\Pi$, and so, $T^{-1}\left(\Pi^{\prime}\right)=\Pi$ [R.2]. Since $T$ is onto, one now has $T(\Pi)=T\left(T^{-1}\left(\Pi^{\prime}\right)\right)=\Pi^{\prime}$. This proves the lemma in the case $k=n-1$. Next, replacing $\mathcal{A}$ and $\mathcal{A}^{\prime}$ by $\Pi$ and $\Pi^{\prime}$, respectively, one analogously proves the lemma for $k=n-2$, if $n \geq 2$. It is now seen that the lemma follows by induction.

Lemma 5 (parallelogram-onto-parallelogram). $T$ maps every parallelogram in $\mathcal{A}$ onto a parallelogram.

Proof. Let $\ell_{1}$ and $\ell_{2}$ be any two parallel lines in $\mathcal{A}$. By Lemma 4 ( $k$-plane-onto$k$-plane), $\ell_{1}^{\prime}:=T\left(\ell_{1}\right)$ and $\ell_{2}^{\prime}:=T\left(\ell_{2}\right)$ are lines in $\mathcal{A}^{\prime}$ and, w.l.o.g., $n^{\prime}=n=2$. Hence, it suffices to show that $\ell_{1}^{\prime} \cap \ell_{2}^{\prime}=\emptyset$. By Lemma 3 (preimage-of- $(n-1)$-planeis- $(n-1)$-plane), $\ell_{1}=T^{-1}\left(\ell_{1}^{\prime}\right)$ and $\ell_{2}=T^{-1}\left(\ell_{2}^{\prime}\right)$. Now, since $T$ is onto, one has $\ell_{1}^{\prime} \cap \ell_{2}^{\prime}=T\left(T^{-1}\left(\ell_{1}^{\prime} \cap \ell_{2}^{\prime}\right)\right)=T\left(T^{-1}\left(\ell_{1}^{\prime}\right) \cap T^{-1}\left(\ell_{2}^{\prime}\right)\right)=T\left(\ell_{1} \cap \ell_{2}\right)=\emptyset$.

Now one is ready to complete the proof of Theorem (main). Let $\left\{P_{0}, P_{1}, P_{2}\right\}$ be a 2 -simplex in $\mathcal{A}$, and $P_{3}:=P_{1}+P_{2}-P_{0}$. Then $\left(P_{0}, P_{1}, P_{2}, P_{3}\right)$ is a parallelogram, and so, by Lemma 5 (parallelogram-onto-parallelogram), $\left(P_{0}^{\prime}, P_{1}^{\prime}, P_{2}^{\prime}, P_{3}^{\prime}\right)$ is a parallelogram, whence $\left\{P_{0}^{\prime}, P_{1}^{\prime}, P_{2}^{\prime}\right\}$ is a 2 -simplex. Therefore, by Lemma 4 ( $k$-planeonto- $k$-plane), there exist [unique] functions $f$ and $g$ from $\mathbb{D}$ onto $\mathbb{D}^{\prime}$ such that for all $\alpha$ and $\beta$ in $\mathbb{D}, T\left(P_{0}+\alpha\left(P_{1}-P_{0}\right)\right)=P_{0}^{\prime}+f(\alpha)\left(P_{1}^{\prime}-P_{0}^{\prime}\right)$ and $T\left(P_{0}+\beta\left(P_{2}-P_{0}\right)\right)=$ $P_{0}^{\prime}+g(\beta)\left(P_{2}^{\prime}-P_{0}^{\prime}\right)$, whence, again by Lemma 5 (parallelogram-onto-parallelogram), $T\left(P_{0}+\alpha\left(P_{1}-P_{0}\right)+\beta\left(P_{2}-P_{0}\right)\right)=P_{0}^{\prime}+f(\alpha)\left(P_{1}^{\prime}-P_{0}^{\prime}\right)+g(\beta)\left(P_{2}^{\prime}-P_{0}^{\prime}\right)$. Note also that $f(0)=0$ and $f(1)=1$. Because the mappings $\mathbb{D}^{2} \ni(\alpha, \beta) \stackrel{e}{\longmapsto} P_{0}+$ $\alpha\left(P_{1}-P_{0}\right)+\beta\left(P_{2}-P_{0}\right)$ and $\mathbb{D}^{\prime 2} \ni\left(\alpha^{\prime}, \beta^{\prime}\right) \stackrel{e^{\prime}}{\longmapsto} P_{0}^{\prime}+\alpha^{\prime}\left(P_{1}^{\prime}-P_{0}^{\prime}\right)+\beta^{\prime}\left(P_{2}^{\prime}-P_{0}^{\prime}\right)$ are 1-to1 and affine, the mapping $\mathbb{D}^{2} \ni(\alpha, \beta) \mapsto(f(\alpha), g(\beta))$, coinciding with $\left(e^{\prime}\right)^{-1} \circ T \circ e$, inherits the preservation of parallelograms and the preservation of collinearity properties of $T$.

It remains to show that $f$ is an isomorphism of $\mathbb{D}$ onto $\mathbb{D}^{\prime}$, and $g=f$. Let $(\alpha, \beta) \in \mathbb{D}^{2}$. If $\alpha \neq 0$, then $((0,1),(\beta, 0),(\alpha+\beta, 0),(\alpha, 1))$ is a parallelogram. Hence, $((0,1),(f(\beta), 0),(f(\alpha+\beta), 0),(f(\alpha), 1))$ is also a parallelogram, whence $f(\alpha+\beta)=$ $f(\alpha)+f(\beta)$; if $\alpha=0$, this additivity property follows from $f(0)=0$.

Next, $(0,0),(\beta, 1)$, and $(\alpha \beta, \alpha)$ are three collinear points in $\mathbb{D}^{2}$. Hence, the points $(0,0),(f(\beta), 1)$, and $(f(\alpha \beta), g(\alpha))$ are collinear as well. Therefore, $f(\alpha \beta)=$ $g(\alpha) f(\beta)$; choosing here $\beta=1$ and recalling that $f(1)=1$, one has $f=g$, and so, $f(\alpha \beta)=f(\alpha) f(\beta)$.

Therefore, $g=f$, and $f$ is a homomorphism, and thus an isomorphism, of $\mathbb{D}$ onto $\mathbb{D}^{\prime}$. Theorem (main) is proved.

Proof of Proposition 1. Note that here $\mathcal{A}$ is finite, since $n$ and $\mathbb{D}=\mathbb{Z}_{2}$ are finite. This, together with $n^{\prime} \geq n$ and $T$ being onto, implies that $T$ is 1-to- 1 and $n^{\prime}=n$. We have to show that $T\left(P_{0}+\alpha\left(P_{1}-P_{0}\right)+\beta\left(P_{2}-P_{0}\right)\right)=P_{0}^{\prime}+\alpha\left(P_{1}^{\prime}-P_{0}^{\prime}\right)+\beta\left(P_{2}^{\prime}-P_{0}^{\prime}\right)$ for all $\left(P_{0}, P_{1}, P_{2}\right) \in \mathcal{A}^{3}$ and all $(\alpha, \beta) \in \mathbb{Z}_{2}^{2}$; if $P_{0}, P_{1}, P_{2}$ are not all distinct or $0 \in\{\alpha, \beta\}$, this is immediate, since $(\alpha, \beta) \in \mathbb{Z}_{2}^{2}$. Hence, it suffices to show that $T\left(P_{0}+\left(P_{1}-P_{0}\right)+\left(P_{2}-P_{0}\right)\right)=P_{0}^{\prime}+\left(P_{1}^{\prime}-P_{0}^{\prime}\right)+\left(P_{2}^{\prime}-P_{0}^{\prime}\right)$ given $P_{0}, P_{1}, P_{2}$ are distinct. Then $P_{0}^{\prime}, P_{1}^{\prime}, P_{2}^{\prime}$ must also all be distinct, because $T$ is 1 -to- 1 . In the case of 
$\mathbb{D}^{\prime}=\mathbb{Z}_{2}$, the points $P_{0}^{\prime}, P_{1}^{\prime}, P_{2}^{\prime}$ being distinct means the same as $\left\{P_{0}^{\prime}, P_{1}^{\prime}, P_{2}^{\prime}\right\}$ being a simplex; likewise, $\left\{P_{0}, P_{1}, P_{2}\right\}$ is a simplex. Suppose now that $q \geq 2$ [the case $n=2$ is even easier [R.9]]. Then, in view of Lemma 1 (lowering-plane-preservationdimension), $T$ maps every 2-plane into a 2-plane; hence,

$$
T\left(P_{0}+\left(P_{1}-P_{0}\right)+\left(P_{2}-P_{0}\right)\right)=P_{0}^{\prime}+\mu\left(P_{1}^{\prime}-P_{0}^{\prime}\right)+\nu\left(P_{2}^{\prime}-P_{0}^{\prime}\right)
$$

for some $\mu$ and $\nu$ in $\mathbb{Z}_{2}$. Because $\left\{P_{0}, P_{1}, P_{2}\right\}$ is a simplex, one has $P_{0}+\left(P_{1}-P_{0}\right)$ $+\left(P_{2}-P_{0}\right) \notin\left\{P_{0}, P_{1}, P_{2}\right\}$, and so, $P_{0}^{\prime}+\mu\left(P_{1}^{\prime}-P_{0}^{\prime}\right)+\nu\left(P_{2}^{\prime}-P_{0}^{\prime}\right) \notin\left\{P_{0}^{\prime}, P_{1}^{\prime}, P_{2}^{\prime}\right\}$, since $T$ is 1-to- 1 . Recalling that $\mu$ and $\nu$ are in $\mathbb{Z}_{2}$, one concludes that $\mu=1$ and $\nu=1$.

\section{REMARKS}

Remark 1. If $\Pi=\bigcap_{i} \Pi_{i} \neq \emptyset$, where the $\Pi_{i}$ 's are affine subspaces of $\mathcal{A}$, then $\Pi$ is an affine subspace as well. Indeed, let $P \in \Pi$; then $\Lambda_{i}:=\Pi_{i}-P$ is a linear subspace of $\mathcal{L}$ for every $i$, and so, $\Lambda:=\bigcap_{i} \Lambda_{i}$ is a linear subspace, and $\Pi=P+\Lambda$.

Remark 2. If $\Pi$ is a $k$-plane in $\mathcal{A}$ and $P \in \mathcal{A} \backslash \Pi$, then $\tilde{\Pi}:=\operatorname{aff}(\Pi \cup\{P\})$ is a $(k+1)$-plane. Indeed, let $Q \in \Pi$; then $\Lambda:=\Pi-Q$ and $\tilde{\Lambda}:=\tilde{\Pi}-Q$ are linear subspaces of $\mathcal{L}$, and $\tilde{\Lambda}=\operatorname{aff}(\Lambda \cup\{R\})$, where $R:=P-Q \notin \Lambda$. Hence, $\operatorname{dim} \tilde{\Pi}=\operatorname{dim} \tilde{\Lambda}=1+\operatorname{dim} \Lambda=k+1$.

Remark 3. If $\Pi_{1}$ and $\Pi_{2}$ are $k$-planes in $\mathcal{A}$ and $\Pi_{1} \neq \Pi_{2}$, then $\operatorname{dim}\left(\Pi_{1} \cap \Pi_{2}\right) \leq k-1$ [R.1, R.2].

Remark 4. If $0 \in \mathcal{A}$, $\operatorname{dim} \Pi=n-1$, and $R \notin \operatorname{Tan} \Pi$, then $\exists \alpha \in \mathbb{D} \alpha R \in \Pi$. Indeed, if $P \in \Pi$ and $\Lambda:=\Pi-P=\operatorname{Tan} \Pi$, then $-P \in \mathcal{A}=\mathbb{D} R+\Lambda$ since $\operatorname{dim} \Lambda=n-1$; hence, $0 \in \mathbb{D} R+\Pi$.

Remark 5. If $\Pi$ and $\Pi_{1}$ are parallel $k$-planes in $\mathcal{A}$, then Tan $\Pi=$ Tan $\Pi_{1}$. Indeed, w.l.o.g., $\operatorname{dim} \mathcal{A}=k+1$ and $0 \in \mathcal{A}$. Put $\Lambda:=\operatorname{Tan} \Pi$ and $\Lambda_{1}:=\operatorname{Tan} \Pi_{1}$ and suppose that, on the contrary, $\Lambda \neq \Lambda_{1}$. Then $\exists R \in \Lambda_{1} \backslash \Lambda$ and $\exists S \in \Lambda \backslash \Lambda_{1}$ [R.3]; hence, $\exists \alpha \in \mathbb{D} \alpha R \in \Pi$ and $\exists \beta \in \mathbb{D} \beta S \in \Pi_{1}$ [R.4]. Then $\alpha R+\beta S \in \Pi+\Lambda=\Pi$; similarly, $\alpha R+\beta S \in \Pi_{1}$; this contradicts $\Pi \cap \Pi_{1}=\emptyset$.

Remark 6. If $\Pi$ and $\Pi_{1}$ are two parallel $(n-1)$-planes, $P \notin \Pi$, and $Q \in \Pi$, then $P Q \cap \Pi_{1} \neq \emptyset$. Indeed, w.l.o.g., $0 \in \mathcal{A}$. Note that $P-Q \notin \operatorname{Tan} \Pi$ [otherwise, $P \in Q+\operatorname{Tan} \Pi \subseteq \Pi+\operatorname{Tan} \Pi=\Pi]$. Hence, $P-Q \notin \operatorname{Tan} \Pi_{1}=\operatorname{Tan}\left(\Pi_{1}-Q\right)[$ R.5]. Therefore, $\exists \alpha \in \mathbb{D} \alpha(P-Q) \in \Pi_{1}-Q$ [R.4], and so, $Q+\alpha(P-Q) \in \Pi_{1} \cap P Q$.

Remark 7. If $P_{0} P_{1} \| P_{2} P_{3}$ and $P_{0} P_{3} \| P_{1} P_{2}$, then $\left(P_{0}, P_{1}, P_{2}, P_{3}\right)$ is a parallelogram. Indeed, $P_{3}-P_{2}=\alpha\left(P_{1}-P_{0}\right)$ and $P_{2}-P_{1}=\beta\left(P_{3}-P_{0}\right)$ for some $\alpha$ and $\beta$ in $\mathbb{D}\left[\right.$ R.5]. Hence, $\left(P_{3}-P_{0}\right)-\left(P_{1}-P_{0}\right)=\alpha\left(P_{1}-P_{0}\right)+\beta\left(P_{3}-P_{0}\right)$. By the linear independence of $P_{1}-P_{0}$ and $P_{3}-P_{0}$, one now has $\beta=1$, whence $P_{2}-P_{1}=P_{3}-P_{0}$.

Remark 8. It is easy to see that a non-empty subset $\Pi$ of $\mathcal{A}$ is an affine subspace iff $P_{0}+\alpha\left(P_{1}-P_{0}\right)+\beta\left(P_{2}-P_{0}\right) \in \mathcal{A}$ for all $\left(P_{0}, P_{1}, P_{2}\right) \in \mathcal{A}^{3}$ and all $(\alpha, \beta) \in \mathbb{D}^{2}$. Let us call a non-empty subset $\Pi$ of $\mathcal{A}$ a quasi-affine subspace if $P_{0}+\alpha\left(P_{1}-P_{0}\right) \in \Pi$ for all $\left(P_{0}, P_{1}\right) \in \Pi^{2}$ and all $\alpha \in \mathbb{D}$; in other words, $\Pi$ is a quasi-affine subspace if for any two distinct points $P_{0}$ and $P_{1}$ of $\Pi$, the line $P_{0} P_{1}$ is in $\Pi$. Accordingly, let us say that $T$ is quasi-semi-affine if there exists an isomorphism $f: \mathbb{D} \rightarrow \mathbb{D}^{\prime}$ such that $T\left(P_{0}+\alpha\left(P_{1}-P_{0}\right)\right)=P_{0}^{\prime}+f(\alpha)\left(P_{1}^{\prime}-P_{0}^{\prime}\right)$ for all $\left(P_{0}, P_{1}\right) \in \mathcal{A}^{2}$ and all $\alpha \in \mathbb{D}$. In other words, $T$ is quasi-semi-affine if there exists an isomorphism $f: \mathbb{D} \rightarrow \mathbb{D}^{\prime}$ 
such that the restriction of $T$ to any line in $\mathcal{A}$ is $f$-semi-affine. Let us say that $T$ is quasi-affine if the restriction of $T$ to any line in $\mathcal{A}$ is affine.

Unless $\mathbb{D}=\mathbb{Z}_{2}$, each of these quasi-notions means exactly the same as the corresponding bona fide notion. This follows because for any $\nu \in \mathbb{D} \backslash\{0,1\}$, one has

$$
P_{0}+\alpha\left(P_{1}-P_{0}\right)+\beta\left(P_{2}-P_{0}\right)=Q_{0}+\beta \lambda^{-1}\left(Q_{2}-Q_{0}\right),
$$

where $Q_{0}:=P_{0}+\alpha\left(P_{1}-P_{0}\right), Q_{2}:=P_{2}+\mu\left(Q_{1}-P_{2}\right), Q_{1}:=P_{0}+\nu\left(Q_{0}-P_{0}\right)$, $\lambda:=1-\nu^{-1}, \mu:=\nu^{-1}$. [For the fields $\mathbb{D}$ of characteristic $\neq 2$, the statement that every quasi-affine subspace is affine can be found in [8, Theorem 5, page 342], where the simpler identity $P+(Q-P)+(R-P)=P+2\left(\left(Q+2^{-1}(R-Q)\right)-P\right)$ is used. It is claimed in [8], though, that the statement is false if $\mathbb{D}$ is of characteristic 2.]

Remark 9 . Suppose here that $\mathbb{D}=\mathbb{Z}_{2}$; then the lines in $\mathcal{A}$ are simply two-point sets, and so, any non-empty subset of $\mathcal{A}$ is a quasi-affine subspace and any $T$ is a quasi-semi-affine mapping, which is even quasi-affine if $\mathbb{D}^{\prime}=\mathbb{D}$. Suppose now that, in addition, $n \leq 2$. Then it is easy to check that every non-empty subset of $\mathcal{A}$ is an affine subspace; respectively, if $T$ is onto and $n^{\prime} \geq n$, then $T$ is 1 -to- 1 and semi-affine.

Remark 10. The line-onto-line condition is needed in Theorem P1 only to assure that the homomorphism $f$ in its proof is onto. This condition can thus be relaxed to the line-into-line one if, say, $\mathbb{D}^{\prime}=\mathbb{D}=\mathbb{Q}, \mathbb{R}$, or $\mathbb{Z}_{p}$ for some prime $p$, in which case, moreover, neither $n$ nor $n^{\prime}$ needs to be assumed finite, and $T$ is then simply affine. Alternatively, one could replace the line-onto-line condition in Theorem P1 by the following set: $n^{\prime} \geq n$ and $T$ is onto and maps every line in $\mathcal{A}$ into a line. Indeed, suppose that $n^{\prime} \geq n$ and $T$ is onto and $\tilde{f}$-semi-affine, where $\tilde{f}: \mathbb{D} \rightarrow f(\mathbb{D})$, $\tilde{f}(\alpha):=f(\alpha) \forall \alpha \in \mathbb{D}$, and $f: \mathbb{D} \rightarrow \mathbb{D}^{\prime}$ is a homomorphism, and so, is an embedding. Then $n \leq n^{\prime}=\operatorname{dim}_{\mathbb{D}^{\prime}} T(\mathcal{A}) \leq \operatorname{dim}_{f(\mathbb{D})} T(\mathcal{A}) \leq \operatorname{dim}_{\mathbb{D}} \mathcal{A}=n$, whence $f(\mathbb{D})=\mathbb{D}^{\prime}$, and so, $T$ is $f$-semi-affine; here, $\operatorname{dim}_{\mathbb{D}}$ stands for the dimension over $\mathbb{D}$.

Remark 11. Condition $\mathbb{D} \neq \mathbb{Z}_{2}$ is essential, according to Remark 12 . The essentiality of $q \in\{1, \ldots, n-1\}$ and of $T$ mapping $q$-planes into $q$-planes is obvious. That of $n^{\prime} \geq n$ and $T$ being onto follows because for any $\mathbb{D} \neq \mathbb{Z}_{2}$, there exists a mapping $h: \mathbb{D} \rightarrow \mathbb{D}$ that is not semi-affine; e.g., for any $\beta \in \mathbb{D} \backslash\{0,1\}$, one may put $h(\beta)=h(0)=0$ and $h(1)=1$. Then $h$ is not semi-affine, because otherwise there exists an automorphism $f$ of $\mathbb{D}$ such that $h(0+\alpha(1-0))=h(0)+f(\alpha)(h(1)-h(0))$ for all $\alpha \in \mathbb{D}$, whence $h=f$, which contradicts the injectivity of $f$. Take then $T$ to be, e.g., the composition of $h$ with the projection of $\mathbb{D}^{n}$ onto $\mathbb{D}$.

[If card $\mathbb{D} \geq 5$, then, moreover, the mapping $h$ just above can be chosen to be bijective; cf. Remark 2 on page 88 in [1]. Indeed, if card $\mathbb{D} \geq 5$, then there exists some $\beta \in \mathbb{D}$ such that $\beta(\beta-1)\left(\beta^{2}-\beta+1\right) \neq 0$; let $h(0)=1, h(1)=0$, and $h(\alpha)=\alpha$ for all $\alpha \in \mathbb{D} \backslash\{0,1\}$. If $h$ were $f$-semi-affine for some automorphism $f$ of $\mathbb{D}$, then $\beta=h(\beta)=h(0+\beta(1-0))=h(0)+f(\beta)(h(1)-h(0))=1-\gamma$, where $\gamma:=f(\beta)$. Similarly, $\beta=h(\beta)=h\left(1-\beta\left(\beta^{-1}-1\right)\right)=-\gamma \beta^{-1}$; eliminating now $\gamma$ from the equations $\beta=1-\gamma$ and $\beta=-\gamma \beta^{-1}$, one would have $\beta^{2}-\beta+1=0$, a contradiction.]

It is easy to understand why the restriction $\mathbb{D} \neq \mathbb{Z}_{2}$ is not needed in "projective" theorems such as Theorems P and P1 above, in contrast with their "affine" counterparts. One explanation is that the additional condition of preservation of parallelism is there more or less implicitly in "projective" theorems, which takes care of $\mathbb{D}=\mathbb{Z}_{2}$. Another related viewpoint is that the "points" of the projective space 
are lines in the underlying linear space, and the collinearity of the "points" means that the corresponding lines are coplanar. Thus, the preservation of the projective version of collinearity under a transformation may be thought of as preservation of 2-planes of the underlying linear space; cf. Corollary 3, where the additional condition $q \geq 2$ allows one to remove the restriction $\mathbb{D} \neq \mathbb{Z}_{2}$. This discussion also shows that the case $q=2$ in Theorem (main) is almost as interesting as $q=1$; it is then rather natural to consider the other values of $q$.

Remark 12. If $\mathbb{D}=\mathbb{Z}_{2}$ and $n \geq 3$, let, w.l.o.g., $\mathcal{A}=\mathbb{Z}_{2}^{n}$; then the set $\Pi:=$ $\left\{\left(\alpha_{1}, \ldots, \alpha_{n}\right) \in \mathbb{Z}_{2}^{n}: \alpha_{n}=0\right\} \cup\{(0, \ldots, 0,1)\} \backslash\{(0, \ldots, 0)\} \subseteq \mathbb{Z}_{2}^{n}$ is not an affine subspace and the mapping $T: \mathbb{Z}_{2}^{n} \rightarrow \mathbb{Z}_{2}^{n}$ that interchanges $(0, \ldots, 0,1)$ and $(0, \ldots, 0)$ and does not move any other points of $\mathbb{Z}_{2}^{n}$ is not semi-affine, while being onto, 1-to-1, and mapping every line onto a line. This remark complements Remarks 8 and 11 .

Remark 13. If $\mathbb{D} \neq \mathbb{Z}_{2}$ and $T(\operatorname{aff}\{P, Q\}) \subseteq$ aff $\{T(P), T(Q)\}$ for any $P$ and $Q$, then for any $k$-plane $\pi$ in $\mathcal{A}$, any $k$, any $P_{0} \in \pi$, and any $P$, one has

$$
T(\operatorname{aff}(\pi \cup\{P\}))=T\left(P_{0}+\left(\pi-P_{0}\right)+\mathbb{D}\left(P-P_{0}\right)\right) \subseteq \operatorname{aff}(T(\pi) \cup\{T(P)\}),
$$

by the identity $\left(^{*}\right)$ of Remark 8, whence, by induction, $T$ (aff $\left.\mathcal{E}\right) \subseteq$ aff $T(\mathcal{E}) \forall \mathcal{E} \subseteq \mathcal{A}$. Therefore, $T$ maps every $k$-plane into a $k$-plane, $\forall k$. Moreover, the image $T(\mathcal{E})$ of any $k$-simplex $\mathcal{E}$ in $\mathcal{A}$ is a $k$-simplex. [Indeed, for any $k$-simplex $\mathcal{E}$ in $\mathcal{A}$, there is an $n$-simplex $\mathcal{F} \supseteq \mathcal{E}$ in $\mathcal{A}$. If $T(\mathcal{E})$ is not a $k$-simplex, then $T(\mathcal{F})$ is not an $n$-simplex, and so, $n-1 \geq \operatorname{dim} T(\mathcal{F})=\operatorname{dim} \operatorname{aff} T(\mathcal{F}) \geq \operatorname{dim} T(\operatorname{aff} \mathcal{F})=\operatorname{dim} T(\mathcal{A})=n^{\prime} \geq n$, a contradiction.] Therefore, $T$ is 1 -to- 1 and maps every $k$-plane onto a $k$-plane. Thus, w.l.o.g., $n=2$, and $T$ maps any two parallel lines $\ell_{1}$ and $\ell_{2}$ in $\mathcal{A}$ onto two parallel lines, because $T\left(\ell_{1}\right) \cap T\left(\ell_{2}\right)=T\left(\ell_{1} \cap \ell_{2}\right)=T(\emptyset)=\emptyset$. It remains to apply Theorem P1; see also the last sentence preceding the statement of Theorem P1.

Remark 14. For any $\mathcal{E} \subseteq \mathcal{A}$ with $\operatorname{dim} \mathcal{E} \leq k$, there exist $P_{0}, \ldots, P_{k}$ in $\mathcal{E}$ such that aff $\mathcal{E}=\left\{\alpha_{0} P_{0}+\cdots+\alpha_{k} P_{k}: \alpha_{0}+\cdots+\alpha_{k}=1, \alpha_{i} \in \mathbb{D} \forall i\right\} ;$ this follows because $\alpha_{0}+\cdots+\alpha_{k}=1$ implies $\alpha_{0} P_{0}+\cdots+\alpha_{k} P_{k}=P_{0}+\alpha_{1}\left(P_{1}-P_{0}\right)+\cdots+\alpha_{k}\left(P_{k}-P_{0}\right)$.

Remark 15. It is easy to see that if $R \in P Q$ and $R \neq Q$, then $P \in R Q$.

Remark 16. If $\mathcal{E}_{1}$ and $\mathcal{E}_{2}$ are subsets of $\mathcal{A}$ and $\mathcal{E}_{1} \cap \mathcal{E}_{2} \neq \emptyset$, then $\operatorname{dim}\left(\mathcal{E}_{1} \cup \mathcal{E}_{2}\right) \leq$ $\operatorname{dim} \mathcal{E}_{1}+\operatorname{dim} \mathcal{E}_{2}$. Indeed, let $\Pi_{1}:=\operatorname{aff} \mathcal{E}_{1}$ and $\Pi_{2}:=$ aff $\mathcal{E}_{2}$. Then there exists $P \in \Pi_{1} \cap \Pi_{2}$; let $\Lambda_{i}:=\Pi_{i}-P$. It follows that $\Pi_{1} \cup \Pi_{2}=\left(\Lambda_{1} \cup \Lambda_{2}\right)-P$, whence $\operatorname{dim}\left(\mathcal{E}_{1} \cup \mathcal{E}_{2}\right) \leq \operatorname{dim}\left(\Pi_{1} \cup \Pi_{2}\right)=\operatorname{dim}\left(\Lambda_{1} \cup \Lambda_{2}\right) \leq \operatorname{dim} \Lambda_{1}+\operatorname{dim} \Lambda_{2}=\operatorname{dim} \Pi_{1}+\operatorname{dim} \Pi_{2}=$ $\operatorname{dim} \mathcal{E}_{1}+\operatorname{dim} \mathcal{E}_{2}$.

\section{REFERENCES}

1. E. Artin, Geometric Algebra, Interscience Publishers, New York, 1957. MR 18:553e

2. W. Benz, Geometrische Transformationen unter besonderer Berücksichtigung der Lorentztransformationen, BI-Wissenschaftsverlag, Mannheim, 1992. MR 93i:51002

3. M. Berger, Geometry I, 1994 Corrected Second Printing, Springer, New York, 1987. MR 88a:51001a

4. H. S. M. Coxeter, The Real Projective Plane, McGraw-Hill, New York, 1949. MR 10:729b

5. J. Frenkel, Géométrie pour l'élève-professeur, Hermann, Paris, 1973.

6. Loo-keng Hua, A theorem on matrices over sfield and its applications, Loo-keng Hua Selected Papers, Springer, New York, 1983, pp. 528-581. MR 84m:01045

7. J. A. Lester, Distance preserving transformations, Handbook of Incidence Geometry, NorthHolland, Amsterdam, 1995, pp. 921-944. MR 96j:51019 
8. A. I. Mal'tsev, Foundations of Linear Algebra, 4th ed., Nauka, Moscow, 1975. MR 11:412h (1948 edition)

9. B. R. McDonald, Geometric Algebra over Local Rings, Marcel Dekker, New York, 1976. MR 57:16198

Cimatron Ltd., Gush Etzion 11, Givat Shmuel, 54030, Israel

E-mail address: sasha@cimatron.co.il

Department of Mathematical Sciences, Michigan Technological University, HoughTON, Michigan 49931

E-mail address: ipinelis@math.mtu.edu 